\title{
Addendum: Plasmonic nanosensors with inverse sensitivity by means of enzyme-guided crystal growth
}

\author{
Laura Rodríguez-Lorenzo, Roberto de la Rica, Ramón A. Álvarez-Puebla, Luis M. Liz-Marzán and Molly M. Stevens
}

Nature Materials 11, 604-607 (2012); published online 27 May 2012; corrected after print 15 December 2017.

Prompted by queries on the potential mechanism for the inverse sensitivity of the biosensing assay reported in the published Letter, as well as about the protocols that we used to obtain the results, here we provide extended discussion of the signal-generation mechanism as well as additional methodological details.

\section{Signal-generation mechanism}

As described in the published Letter, the signal is generated by the reduction of silver ions on gold nanostars. The reduction of silver triggers a shift in the localized surface plasmon resonance (LSPR) of the dispersed gold nanostars (which act as sensors). The reduction of silver on the gold nanostars is known to be affected by the following factors:

(i) The concentration of hydrogen peroxide generated by glucose oxidase (GOx), which can reduce silver ions ${ }^{1}$.

(ii) The concentration of ammonia, which dictates the $\mathrm{pH}$ of the solution (it is well established that the reduction potential of a chemical species depends on the $\mathrm{pH}$ of the solution ${ }^{2}$. Also, ammonia coordinates silver ions, thereby changing their reduction potential ${ }^{3}$ ).

(iii) The presence of glucose, which is a weak reducing agent and is found in vast excess ${ }^{4}$.

(iv) The presence of 2 -( $N$-morpholino)ethanesulfonic acid (MES), which can also act as a weak reducing agent ${ }^{5}$.

(v) The presence of light (it is well known that UV light can reduce silver ions ${ }^{6}$ ).

(vi) The concentration of poly(vinylpyrrolidone) (PVP) around the nanostars. PVP can block the gold surface and therefore change the reduction potential of silver ions on gold. Also, PVP has also been shown to act as a weak reducing agent capable of reducing silver ions ${ }^{7}$. Furthermore, PVP can coordinate silver ions. The nitrogen and oxygen atoms in the pyrrolidyl groups of PVP molecules may donate their lone unpaired electron to $s p$ hybrid orbitals of $\mathrm{Ag}^{+}$. Two kinds of coordination bond (PVP molecular intra- and inter-chain interactions) can take place ${ }^{8}$, which may significantly decrease the chemical potential and further facilitate the reduction of PVP-attached silver ions to generate a silver coating around the nanostars ${ }^{9}$. The reduction of silver ions on gold nanoparticles can also be affected by the presence of PVP because PVP can selectively adsorb silver nanostructures. Indeed, in gold-silver core-shell nanoparticles with cubic, triangularbipyramidal, and rod or wire silver shells, $\{100\}$-type dominant facets were formed upon epitaxial growth on the $\{111\}$-type facets of the gold core ${ }^{10,11}$. The morphology change between the Au core and Ag shells may have its origin in the change of adsorption selectivity of PVP molecules from $\mathrm{Au}\{111\}$-type facets to $\mathrm{Ag}\{100\}$-type ones in ethylene glycol solvent. Such selective adsorption of PVP molecules can also affect the growth rate of the adsorbed Ag facets. Finally, it should be noted that the PVP around the nanostars may play a crucial role in other processes not related to the growth of silver nanoparticles. For example, the reshaping of nanostars depends on the concentration of PVP around them ${ }^{12}$. Moreover, different batches of nanostars may have slightly different LSPRs, owing to slightly different concentrations of PVP around these colloids. Since the concentration of PVP around the nanostars depends on the number of washing steps to which the nanostars are subjected, the LSPR of the nanostars may also change depending on the number of intermediate steps preceding the silver reduction on the nanostars. For all these reasons, we reported changes in the LSPR of the nanostars resulting from the reduction of silver ions on gold as a shift with respect to a blank experiment performed in the same conditions and with nanostars obtained from the same batch.

(vii) The presence of unreacted aldehyde groups around the nanostars. Aldehydes have been previously used to reduce metal ions on nanoparticle seeds ${ }^{13}$. Unreacted aldehyde groups did not affect the reported data (Fig. 4) because they were blocked with bovine serum albumin (BSA) and ethanolamine to avoid non-specific interactions in the immunoassay. However, aldehyde groups were not blocked in the experiments shown in Fig. 2c, because no biorecognition experiments were performed with the nanostars bearing covalently bound GOx.

Our experiments were performed in excess of glucose, MES and light. We observed that the reaction did not take place (to an extent that would have been observable) in the absence of GOx when aldehyde groups were blocked (blue dots in Fig. 4a and green dots in Fig. 4b), because glucose, MES and light are weak reducing agents. Background signal in the presence of glucose (Fig. 2c, red dots) may have resulted from the reduction of $\mathrm{Ag}^{+}$by unblocked aldehyde groups, even in the absence of GOx, as observed when GOx was diluted down to $10^{-20} \mathrm{~g} \mathrm{~mL}^{-1}$ (at this concentration we would expect the possibility of less than one molecule of enzyme to be present in the solution). However, as the concentration of GOx increases, the effects on the signal from the biocatalytic production of hydrogen peroxide are much stronger than those from unblocked aldehydes. Background signal is not present in the absence of glucose (Fig. 2c, black dots). And for the actual assay (Fig. 4) there is no background signal because aldehyde groups were blocked, and therefore $\mathrm{Ag}^{+}$cannot be reduced in the absence of the hydrogen peroxide produced by GOx (as is evidenced by the fact that the shift falls to zero when there is no prostatespecific antigen (PSA), and by the absence of a shift when BSA is substituted for PSA). Please note that for Supplementary Fig. S5 the gold nanostars were not modified with GOx and therefore also did not have glutaraldehyde added. The reduction of silver ions on gold, even in the absence of GOx, is also affected by the concentration of ammonia and by the concentration of PVP around the nanostars. For example, silver ions may be reduced on gold nanostars by glucose in the absence of GOx when the concentration of ammonia is much higher than the $40 \mathrm{mM}$ concentration reported, because the reduction potentials of glucose and silver ions are different at higher $\mathrm{pH}^{\mathrm{Halues}}{ }^{14}$.

Hydrogen peroxide is a stronger reducing agent than glucose, MES and light. This means that hydrogen peroxide can reduce silver ions on gold in the presence of low concentrations of ammonia. In the signal-generation mechanism proposed in the Letter, the production of a low concentration of hydrogen peroxide by GOx triggers the reduction of silver ions on gold. In other words, a small concentration of hydrogen peroxide can overcome the energy barrier required to reduce silver ions on gold. Once this energy barrier is overcome, the system has enough reactive species to continue reducing silver ions, since there is an excess of glucose, MES and light. Therefore, a small concentration of enzyme-generated hydrogen peroxide can trigger the formation of a silver coating around gold nanostars. This phenomenon is likely responsible for the ultralow limit of detection reported in the Letter.

The inverse-sensitivity phenomenon is related to the kinetics of metal reduction. It is generally accepted that fast kinetics of reduction 
favour nanoparticle nucleation. In other words, they favour the formation of new nanoparticles. Instead, slow kinetics of metal reduction favour the reduction of silver ions on pre-existing nanoparticles. This results in the formation of larger nanoparticles. That is, slow kinetics of metal reduction favour growth over nucleation. The kinetics of reduction of noble metal nanoparticles are influenced, among other factors, by:

(i) The reduction potential of the reducing agent as well as any factor affecting this reduction potential (such as the $\mathrm{pH}$ of the solution, and the presence of capping ligands or chelating agents) ${ }^{15}$.

(ii) The temperature of the solution ${ }^{16}$.

(iii) The concentration of the reducing agent. Higher concentrations of reducing agent lead to faster kinetics of metal reduction ${ }^{17}$.

In our experiments, the $\mathrm{pH}$ was buffered, the concentration of ammonia fixed, the temperature constant, and variations in the concentration of the weak reducing agent glucose are irrelevant. Therefore, the key factor that governs the kinetics of crystal growth is the concentration of the stronger reducing agent hydrogen peroxide. When the concentration of hydrogen peroxide is low (near the limit of detection), the kinetics of reduction are slow. This favours growth over nucleation, and the silver ions are reduced as a coating around the nanostars. The silver coating generates a blue shift in the LSPR of the gold nanostars, which is the basis of the detection mechanism described in the Letter. When the concentration of hydrogen peroxide increases, the kinetics of reduction is fast. This favours nucleation over growth, and separate silver nanoparticles are formed (it is important to stress that the assay measures changes in the LSPR of the nanostars; separate silver nanoparticles do not change the LSPR of the nanostars but can be detected with TEM). Therefore, faster kinetics of reduction lead to smaller LSPR shifts because less silver is deposited as a coating around the nanostars. This relationship between the concentration of hydrogen peroxide and the LSPR shift enables the analyst to quantify the levels of the target molecule, since its concentration is related to the concentration of peroxide through the biocatalytic cycle of GOx bound to the nanostars. The sensitivity of an analytical method is usually defined as the slope of the calibration curve, that is, the change in signal registered for a particular change in the concentration of the analyte. In our case, the slope of the calibration curve is negative, and therefore the sensitivity is inversed when compared to other analytical methods with a positive calibration curve. Negative slopes are not uncommon in analytical chemistry; for example, they can be found in competitive immunoassays. Our method is different from these approaches in that the highest signal possible (larger difference between the signal of the analyte and the blank signal) is registered at the lowest concentration of analyte within the inverse sensitivity regime.

Noise levels in Fig. 4a,b were shown as error bars that represent the standard deviation of three measurements performed on three different samples. This is standard methodology for variability measurements in analytical chemistry ${ }^{18-20}$. The origin of the error may depend on a large number of variables (dilution error, analyst proficiency, pipette calibration, instrumental error and many others). Figure 4a,b shows a log-linear response between analyte concentration and the blueshift of the LSPR; that is, the LSPR blueshift varies with the logarithm of the number of molecules. As a consequence, variability in the concentration of PSA (horizontal axis), including that attributable to Poisson sampling, would not be expected to result in proportionate variation in the phase shift (vertical axis). Such changes (or uncertainties) in the number of molecules will correspond to substantially smaller changes in the blueshift, as observed in the data reported in the Letter (it should be noted that the only data for which noise is relevant is those in Fig. 4, as these experiments represent the actual performance for the assay and were carried out in triplicate).

\section{Detailed protocols}

The detection of PSA in Fig. 4 was carried out by following these protocols.

Modification of gold nanostars with polyclonal anti-PSA. Gold nanostars (NSs) were synthesized according to a previously published method with a modification in the molecular weight of PVP $\left(M_{\mathrm{w}}=8,000 \text {, Alfa-Aesar } 41626\right)^{21}$. After removing excess layers of PVP by centrifugation and redispersion in isopropanol $(2,950 \times g, 3$ times), the concentrations of gold and PVP were $0.80 \mathrm{mM}$ and $3 \mathrm{mM}$, respectively.

$7 \mathrm{~mL}$ of NSs $\left([\mathrm{Au}] \sim 0.80 \mathrm{mM}\right.$ ) was centrifuged at $2,950 \times g$ for $30 \mathrm{~min}$ at $25^{\circ} \mathrm{C}$. The NSs were redispersed in $7 \mathrm{~mL}$ sodium bicarbonate buffer solution (10 mM, pH 9). The gold concentration was $\sim 0.70 \mathrm{mM}$. $0.7 \mathrm{~mL}$ of $50 \%$ glutaraldehyde solution (Sigma G7651) were added. The final concentrations of glutaraldehyde and gold were $5 \%$ and $\sim 0.65 \mathrm{mM}$, respectively. The solution was shaken gently (on a roller) for $3 \mathrm{~h}$ at $25^{\circ} \mathrm{C}$. The NSs were then centrifuged at $2,380 \times \mathrm{g}$ for $25 \mathrm{~min}$. The resulting pellet was redispersed in $7 \mathrm{~mL}$ of a sodium bicarbonate buffer solution ( $10 \mathrm{mM}, \mathrm{pH}$ 9). The final gold concentration was $\sim 0.50 \mathrm{mM}$. $20 \mu \mathrm{L}$ of a $1 \mathrm{mg} \mathrm{mL}^{-1}$ anti-PSA solution were added to $\sim 0.50 \mathrm{mM}$ of gold nanostars and $2 \mathrm{mM}$ of $\mathrm{NaCNBH}_{3}$ in sodium bicarbonate buffer $(10 \mathrm{mM}$, pH 9). The final reaction volume was $7 \mathrm{~mL}$. The reaction was allowed to proceed for $3 \mathrm{~h}$ at room temperature. Non-reacted aldehyde groups were blocked by adding 100 $\mu \mathrm{L}$ of a $100 \mathrm{mM}$ ethanolamine solution (bicarbonate buffer $10 \mathrm{mM} \mathrm{pH}$ 9) for $1 \mathrm{~h} .100 \mu \mathrm{L}$ of $1 \mathrm{mg} \mathrm{mL}^{-1} \mathrm{BSA}$ in bicarbonate buffer (10 mM, $\mathrm{pH}$ 9) were then added. The reaction was allowed to proceed overnight at $4{ }^{\circ} \mathrm{C}$. The NSs were centrifuged at $1,450 \times g$ for $20 \mathrm{~min}$. The resulting pellet was redispersed in $7 \mathrm{~mL}$ PBS buffer $(10 \mathrm{mM}, \mathrm{pH} 7.4)$. The final gold concentration was $\sim 0.5 \mathrm{mM}$.

Immunoassay. A PSA stock solution (PBS buffer $10 \mathrm{mM}, \mathrm{pH} 7.4$ ) with the concentration of $10 \mu \mathrm{g} \mathrm{mL}^{-1}$ was serially diluted (1:10). This was done by adding $100 \mu \mathrm{L}$ (using a micropipette) of $10 \mu \mathrm{g} \mathrm{mL}^{-1}$ PSA solution to $900 \mu \mathrm{L}$ of PBS, and then mixing. The rest of the calibrating solutions were prepared in the same way: by adding $100 \mu \mathrm{L}$ of a 10 -fold more concentrated PSA solution to $900 \mu \mathrm{L}$ of PBS.

An appropriate volume of the diluted samples was added to $500 \mu \mathrm{L}$ of anti-PSA-conjugated NSs in order to obtain the desired final concentrations of PSA. The final volume was $1 \mathrm{~mL}$. The dispersions were incubated for 90 min at room temperature. The NSs were centrifuged at $450 \times g$ for $10 \mathrm{~min}$. The resulting pellet was redispersed in $500 \mu \mathrm{L}$ PBS. $10 \mu \mathrm{L}$ of monoclonal anti-PSA antibody were added. The dispersions were incubated for $2 \mathrm{~h}$ at room temperature. The NSs were centrifuged at $450 \times g$ for 10 min. The pellet was redispersed in $500 \mu \mathrm{L}$ of PBS (10 mM, pH 7.4). $10 \mu \mathrm{L}$ of GOx-conjugated anti-mouse IgG were added to each sample. The dispersions were incubated for $2 \mathrm{~h}$ at room temperature. The NSs were centrifuged at $450 \times g$ for $5 \mathrm{~min}$. The pellet was redispersed in $400 \mu \mathrm{L} \mathrm{MES} \mathrm{(10} \mathrm{mM,} \mathrm{pH} 6$ ).

For the reduction of silver, a $1 \mathrm{M}$ glucose solution in MES buffer $(10 \mathrm{mM}, \mathrm{pH}$ 6) was prepared. $100 \mu \mathrm{L}$ of the $1 \mathrm{M}$ glucose solution was added to $400 \mu \mathrm{L}$ NSs in MES buffer. The reaction was allowed to proceed for $1 \mathrm{~h}$ at room temperature. During this period GOx generates hydrogen peroxide.

A fresh solution of $\mathrm{AgNO}_{3}(0.2 \mathrm{mM})$ in $80 \mathrm{mM} \mathrm{NH}$ (aq) was prepared and filtered with a syringe filter $(0.2 \mu \mathrm{m}) .500 \mu \mathrm{L}$ of the resulting $\left[\mathrm{Ag}\left(\mathrm{NH}_{3}\right)_{2}\right]$ solution was added to the mixture of protein-covered NSs and glucose. Visible-near-infrared (vis-NIR) spectra 
were recorded after $2 \mathrm{~h}$. The LSPR shift was calculated with respect to a blank experiment in the absence of the target molecule.

The reduction of silver ions by GOx covalently attached to the nanostars (Fig. 2) was achieved by following the following protocol.

Modification of gold nanostars with glucose oxidase. NSs were synthesized according to a previously published method with a modification in the molecular weight of PVP $\left(M_{\mathrm{w}}=8,000 \text {, Alfa-Aesar 41626 }\right)^{21}$. After removing excess PVP by centrifugation and redispersion in isopropanol $(2950 \times g$, 3 times $)$, the gold and PVP concentrations were $0.85 \mathrm{mM}$ and $\sim 3$ mM, respectively.

$10 \mathrm{~mL}$ of NSs $([\mathrm{Au}]=0.86 \mathrm{mM})$ were centrifuged at $2,950 \times \mathrm{g}$ for $30 \mathrm{~min}$ at $25^{\circ} \mathrm{C}$. The NSs were redispersed in $10 \mathrm{~mL}$ of sodium bicarbonate buffer (10 mM, pH 9). The gold concentration was $0.74 \mathrm{mM} .1 \mathrm{~mL}$ of $50 \%$ glutaraldehyde solution (Sigma G7651) were added to the NS solution. The final concentrations of glutaraldehyde and gold were $5 \%$ and $0.61 \mathrm{mM}$, respectively. The dispersion was then shaken gently (on a roller) for $3 \mathrm{~h}$ at $25^{\circ} \mathrm{C}$. Then the NSs were centrifuged at $1,880 \times g$ for 20 min. The resulting pellet was redispersed in $10 \mathrm{~mL}$ sodium bicarbonate buffer $(10 \mathrm{mM}, \mathrm{pH}$ 9). The final gold concentration was $0.57 \mathrm{mM}$.

A stock solution of $2 \mathrm{mg} \mathrm{mL}^{-1}$ of GOx was prepared in sodium bicarbonate buffer (10 mM, pH 9). The GOx stock solution was serially diluted in sodium bicarbonate buffer $(10 \mathrm{mM}$ pH 9). An appropriate volume of the diluted GOx solutions was added to $0.57 \mathrm{mM}$ gold nanostars and $2 \mathrm{mM} \mathrm{NaCNBH}_{3}$ in the sodium bicarbonate buffer $(10 \mathrm{mM}, \mathrm{pH}$ 9) in order to obtain the desired final enzyme concentration. The final volume was $1 \mathrm{~mL}$. The reaction was let to proceed for $3 \mathrm{~h}$ at room temperature. The resulting protein-modified nanostars were centrifuged at $1,000 \times g$ for $20 \mathrm{~min}$. The resulting pellet was redispersed in $400 \mu \mathrm{L}$ of MES buffer $10 \mathrm{mM}$, pH 6 . The final gold concentration was $0.5 \mathrm{mM}$.

For the reduction of silver, a solution containing $1 \mathrm{M}$ glucose in MES buffer $(10 \mathrm{mM}, \mathrm{pH}$ 6) was prepared. $100 \mu \mathrm{L}$ of a $1 \mathrm{M}$ glucose solution was added to $400 \mu \mathrm{L}$ of $0.5 \mathrm{mM}$ protein-coated NSs in MES buffer for $1 \mathrm{~h}$ at room temperature. The addition of glucose resulted in the production of hydrogen peroxide by the enzyme.

A fresh solution of $\mathrm{AgNO}_{3}(0.2 \mathrm{mM})$ in $80 \mathrm{mM} \mathrm{NH}_{3}(\mathrm{aq})$ was prepared and filtered with a syringe filter $(0.2 \mu \mathrm{m}$ cut-off). It is important

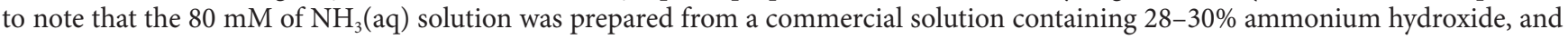
that the $\mathrm{NH}_{3}$ concentration in this solution rapidly changed, owing to evaporation. Therefore, the exact concentration of the $\mathrm{NH}_{3}(\mathrm{aq})$ solution required to coordinate silver ions and to modify the $\mathrm{pH}$ from 6 to 9 in the proposed experimental conditions needs to be optimized. $500 \mu \mathrm{L}$ of the resulting $\left[\mathrm{Ag}\left(\mathrm{NH}_{3}\right)_{2}\right]$ solution was added to the mixture of protein-coated nanostars and glucose. Vis-NIR spectra were recorded after $2 \mathrm{~h}$. The shift in LSPR was calculated with respect to a blank experiment in the absence of GOx.

\section{References}

1. Zhang, Y. et al. A quantitative colorimetric assay of $\mathrm{H}_{2} \mathrm{O}_{2}$ and glucose using silver nanoparticles induced by $\mathrm{H}_{2} \mathrm{O}_{2}$ and UV. Chinese Chem. Lett. 24, 1053-1058 (2013).

2. Bastús, N. G., Merkoçi, F., Piella, J. \& Puntes, V. Synthesis of highly monodisperse citrate-stabilized silver nanoparticles of up to $200 \mathrm{~nm}$ : kinetic control and catalytic properties. Chem. Mater. 26, 2836-2846 (2014).

3. Wang, C., Liu, G., Liu, R., Li, W. \& Zhang, W. Stability improving effect of silver diamminohydroxide precursor in green hydrothermal synthesis of silver nanoparticle colloids. Micro Nano Lett. 9, 320-324 (2014).

4. Tharion, J., Satija, J. \& Mukherji, S. Glucose mediated synthesis of gold nanoshells: A facile and eco-friendly approach conferring high colloidal stability. RSC Adv. 4, 3984 (2014).

5. Lopez, A. \& Liu, J. DNA-templated fluorescent gold nanoclusters reduced by Good's buffer: from blue-emitting seeds to red and near infrared emitters. Can. J. Chem. 93, 615-620 (2015).

6. Darroudi, M., Ahmad, M. B., Zak, A. K., Zamiri, R. \& Hakimi, M. Fabrication and characterization of gelatin stabilized silver nanoparticles under UV-light. Int. J. Mol. Sci. 12, 6346-6356 (2011).

7. Hoppe, C. E., Lazzari, M., Pardiñas-Blanco, I. \& López-Quintela, M. A. One-step synthesis of gold and silver hydrosols using poly( $N$-vinyl-2-pyrrolidone) as a reducing agent. Langmuir 22, 7027-7034 (2006).

8. Wang, H., Qiao, X., Chen, J., Wang, X. \& Ding, S. Mechanisms of PVP in the preparation of silver nanoparticles. Mater. Chem. Phys. 94, 449-453 (2005).

9. Jiang, C., Nie, J. \& Ma, G. A polymer/metal core-shell nanofiber membrane by electrospinning with an electric field, and its application for catalyst support. RSC Adv. 6, 22996-23007 (2016).

10. Tsuji, M. et al. Shape-dependent evolution of Au@Ag core-shell nanocrystals by PVP-assisted N,N-dimethylformamide reduction. Cryst. Growth Des. 8, 2528-2536 (2008).

11. Tsuji, M. et al. crystal structures and growth mechanisms of icosahedral Au@Ag core-shell and Au/Ag twin nanocrystals prepared by PVP-assisted N,Ndimethylformamide reduction. Cryst. Growth Des. 10, 4085-4090 (2010).

12. Rodríguez-Lorenzo, L., Álvarez-Puebla, R. A., García de Abajo, F. J. \& Liz-Marzan, L. M. Surface enhanced Raman scattering using star-shaped gold colloidal nanoparticles. J. Phys. Chem. C 114, 7336-7340 (2010).

13. Brinson, B. E. et al. Nanoshells made easy: improving Au layer growth on nanoparticle surfaces. Langmuir 24, 14166-14171 (2008).

14. Li, T. et al. Sensitive detection of glucose based on gold nanoparticles assisted silver mirror reaction. Analyst 136, 2893-2896 (2011).

15. Fukui, R., Katayama, Y. \& Miura, T. The influence of potential on electrodeposition of silver and formation of silver nanoparticles in some ionic liquids. J. Electrochem. Soc. 158, D567 (2011).

16. Jiang, X. C., Chen, W. M., Chen, C. Y., Xiong S. X. \& Yu, A. B. Role of temperature in the growth of silver nanoparticles through a synergetic reduction approach. Nanoscale Res. Lett. 6, 32 (2011).

17. Piella, J. Bastús, N. G. \& Puntes, V. Size-controlled synthesis of sub-10-nanometer citrate-stabilized gold nanoparticles and related optical properties. Chem. Mater. 28, 1066-1075 (2016).

18. Luo, X., Xu, M., Freeman, C., James, T. \& Davis, J. J. Ultrasensitive label free electrical detection of insulin in neat blood serum. Anal. Chem. 85, 4129-4134 (2013).

19. Averseng, O., Hagège, A., Taran F. \& Vidaud, C. Surface plasmon resonance for rapid screening of uranyl affine proteins. Anal. Chem. 82, 9797-9802 (2010).

20. Zhu, H., Dale, P. S., Caldwell, C. W. \& Fan, X. Rapid and label-free detection of breast cancer biomarker CA15-3 in clinical human serum samples with optofluidic ring resonator sensors. Anal. Chem. 81, 9858-9865 (2009).

21. Kumar, P. S., Pastoriza-Santos, I., Rodríguez-González, B., García de Abajo, F. J. \& Liz-Marzán, L. M. High-yield synthesis and optical response of gold nanostars. Nanotechnology 19, 015606 (2008). 